\title{
Aspectos etnoecológicos e implicações para a conservação de Tursiops truncatus (Cetartiodactyla: Delphinidae) no Arquipélago das Cagarras, Rio de Janeiro, Brasil
}

\author{
Liliane Lodi ${ }^{1}$, Camilah Antunes Zappes ${ }^{2 *} \&$ Álvaro Samuel Guimarães dos Santos ${ }^{1}$ \\ ${ }^{1}$ Faculdades Integradas Maria Thereza, Rio de Janeiro, RJ, Brasil. \\ ${ }^{2}$ Departamento de Geografia de Campos, Instituto de Ciências da Sociedade e Desenvolvimento Regional, \\ Universidade Federal Fluminense, Campos dos Goytacazes, Rio de Janeiro, Brasil.
}

\begin{abstract}
Resumo - Os objetivos deste estudo foram compreender os aspectos ecológicos do golfinho-flíper (Tursiops truncatus) no Arquipélago das Cagarras, Rio de Janeiro, e possíveis impactos antropogênicos através da comparação entre o conhecimento dos pescadores artesanais locais (GA) e dos frequentadores do arquipélago (GB). Os dados obtidos foram comparados com os disponíveis na literatura a fim de propor estratégias de monitoramento e conservação. Entre agosto e dezembro de 2010, foram aplicados os métodos de observação participante, entrevistas-questionários e uso de pranchas ilustrativas. Foram conduzidas 61 entrevistas, das quais $83,6 \%$ foram selecionadas através de respostas corretas sobre a identificação da espécie pela prancha (características morfológicas) e descrição da época de ocorrência no arquipélago (julho a dezembro), como reportada para a espécie na literatura. Das sete questões analisadas estatisticamente, foi encontrada diferença significativa entre os relatos dos dois grupos em relação às ameaças antrópicas a T. truncatus. A rede de emalhe e a diminuição dos recursos pesqueiros foram as principais causas indicadas pelo GA. Já o GB indicou o uso desordenado do espaço, a poluição e a pesca predatória como os fatores que ameaçavam os golfinhos-flíper. O uso de dados etnobiológicos em programas de conservação apresenta oportunidades para a colaboração e participação da comunidade que está diretamente inserida no ecossistema, bem como para o envolvimento de atores locais em questões relacionadas à sustentabilidade do arquipélago.
\end{abstract}

Palavras-chave adicionais: conhecimento local, ecologia, golfinho-flíper, manejo.

\begin{abstract}
Ethnoecological aspects and implications for conservation of Tursiops truncatus (Cetartiodactyla: Delphinidae) in the Cagarras Archipelago, Rio de Janeiro, Brazil) - The objectives of this study were to understand the ecological aspects of the common bottlenose dolphin (Tursiops truncatus) in the Cagarras Archipelago, Rio de Janeiro, Brazil, and possible anthropogenic impacts by comparing the knowledge of local small-scale fishermen (GA) and the regulars in the archipelago (GB). The data obtained were compared with those available in the literature to propose strategies for monitoring and preservation. Between August and December 2010, we applied the methods of participant observation, interviews-questionnaires and use of illustrative posters. Sixty-one interviews were conducted, from which $83.6 \%$ were selected by considering correct answers about the identification of the species on posters (morphological characteristics) and description of time of occurrence in the archipelago (July to December) as reported for the species in the literature. Regarding the seven questions analyzed statistically, a significant difference was found between the accounts of the two groups with respect to anthropic threats to T. truncatus. Gill-nets and the decrease of fishing resources were the main causes identified by the GA group whereas the GB group pointed to the disorganized use of space, pollution and predatory fishing as the factors that have threatened the common bottlenose dolphins. The use of ethnobiological data in conservation programs brings opportunities for the collaboration and participation of the community that is directly inserted into the ecosystem as well as a chance for locals to get involved in issues concerning the sustainability of the archipelago.
\end{abstract}

Additional key words: common bottlenose dolphin, ecology, local knowledge, management.

O golfinho-flíper, Tursiops truncatus (Montagu, 1821) está amplamente distribuído na costa brasileira, ocorrendo desde o Amapá ao Rio Grande do Sul (Barreto 2011). Essa distribuição expõe a espécie a uma ampla variedade de ameaças diretas e indiretas que operam de diferentes formas nas populações locais (e.g., Fruet et al. 2010). Capturas acidentais em artefatos de pesca, degradação do ambiente, tráfego de embarcações e exploração excessiva de recursos importantes na sua dieta, por exemplo, constituem ameaças potenciais à sua conservação. Atualmente, Tursiops truncatus é considerada uma das principais espécies de cetáceos sob forte pressão antrópica no Brasil (IBAMA 2001).

\footnotetext{
*Autora para correspondência: lilianelodi@gmail.com

Editor responsável: Ulysses Paulino de Albuquerque

Submetido em 18 maio 2012; aceito em 5 maio 2013

Publicação inicial: 18 Jul. 2013; versão final: 2 maio 2014
}

Devido à sua proximidade com a costa, o Arquipélago das Cagarras, estado do Rio de Janeiro, é uma área intensamente utilizada pelos pescadores e moradores das cidades do Rio de Janeiro e de Niterói, como área para a realização de atividades de pesca artesanal e esportiva, lazer e recreação (Lodi 2009). Esta proximidade permite que pescadores e frequentadores recreacionais da área observem com frequência os golfinhos-flíper, o que ao longo dos anos contribuiu para a organização e elaboração do conhecimento local sobre a biologia e ecologia da espécie (Zappes et al. 2011a). Esse conhecimento é patrimônio de uma comunidade, construído ao longo dos anos por meio da intuição, percepção e vivência no meio, e é repassado oralmente entre gerações (Begossi 1992; Diegues 2001). No arquipélago, a ocorrência do golfinho-flíper segue um padrão sazonal, entre julho e dezembro (Barbosa et al. 2008; Lodi 2009). 
No Brasil, estudos relacionados à etnoecologia de cetáceos são realizados desde a década de 1990 e abordam principalmente questões relativas à taxonomia, comportamento e interações entre as espécies e as comunidades humanas costeiras (e.g., Simões-Lopes 1991; Pinheiro \& Cremer 2003; Souza $\&$ Begossi 2007; Oliveira et al. 2008; Peterson et al. 2008; Zappes et al. 2009, 2010a,b, 2011a,b). Para o Arquipélago das Cagarras e áreas adjacentes, Zappes et al. (2010a) reportaram as categorias comportamentais de $T$. truncatus e Zappes et al. (2011a) descreveram a ocorrência de interações negativas entre os golfinhosflíper e pescadores artesanais da região. No presente estudo, a abordagem etnoecológica foi utilizada para complementar os trabalhos de Zappes et al. (2010a, 2011a) acerca do conhecimento local das comunidades de pescadores, incluindo também usuários recreacionais do arquipélago, sobre $T$. truncatus.

Neste sentido, os objetivos deste estudo foram: 1- compreender os aspectos ecológicos do golfinhoflíper no Arquipélago das Cagarras, principalmente direcionados ao uso do hábitat, através do conhecimento dos pescadores artesanais e usuários recreacionais que frequentam o local (usuários de diferentes profissões que visitam a região); 2- verificar a percepção desses atores sociais sobre os possíveis impactos antropogênicos; 3- comparar a percepção de conservação da espécie entre pescadores e usuários recreacionais; 4- estabelecer um paralelo entre o conhecimento local dos grupos estudados e confrontar os dados disponíveis na literatura; e 5- propor ações de conservação envolvendo atores locais, instituições de pesquisas, organizações governamentais e não governamentais.

Os dados obtidos neste estudo, juntamente com as informações etnoecológicas pretéritas (Zappes et al. 2010a, 2011a), poderão nortear estratégias de monitoramento para o manejo de $T$. truncatus e propostas de conservação do arquipélago que serão futuramente implementadas através do Plano de Manejo do Monumento Natural do Arquipélago das Ilhas Cagarras, Unidade de Conservação criada em abril de 2010 (Lei Federal No 12.229).

\section{MATERIAL e Métodos}

Área de estudo. O Arquipélago das Cagarras (230.' $\left.50^{\prime \prime} \mathrm{S}, 43^{\circ} 12^{\prime} 70^{\prime \prime} \mathrm{W}-23^{\circ} 03^{\prime} 00^{\prime \prime} \mathrm{S}, 43^{\circ} 12^{\prime} 50^{\prime \prime} \mathrm{W}\right)$ é um pequeno grupo de ilhas costeiras, localizado cerca de 3,8 km da Ponta do Arpoador, Praia de Ipanema, estado do Rio de Janeiro, sudeste do Brasil (Figura 1). Trata-se de um conjunto formado por três ilhas principais (da Cagarra Grande, de Palmas e Comprida), Ilhota Filhote da Cagarra e Lajes Matias e Praça Onze. (Moraes et al. 2013). Apesar de seu valor científico e econômico e da proximidade com um dos maiores centros urbanos do país, a cidade do Rio de Janeiro, o levantamento biológico do arquipélagp foi realizado apenas recentemente (Moraes et al. 2013). Sua proximidade física com um grande centro urbano facilita a presença de visitantes e viabiliza a prática da pesca artesanal, já que pescadores não precisam se deslocar a grandes distâncias (Rangel et al. 2007). O arquipélago trata-se de um ponto turístico, mas não há moradores, nem qualquer tipo de estrutura em sua área.

Coleta de dados. Foram utilizados os métodos da observação participante (observação direta), que permitiu que os pesquisadores estabelecessem contato direto com os atores locais (Richardson 1999), e entrevistas etnográficas individuais através da aplicação de questionário (Kendall 2008). A comunidade estudada foi subdividida em dois grupos: GA- pescadores artesanais da Colônia de Pescadores Z-8, com aproximadamente 90 pescadores, distribuídos em 32 embarcações (Fundação de Amparo à Pesquisa de Recursos Vivos na Zona Econômica Exclusiva 2005), e que realizam suas operações de pesca no arquipélago e áreas adjacentes; e GB- frequentadores de diversas classes sociais residentes nas cidades do Rio de Janeiro e de Niterói.

Entre agosto e dezembro de 2010, foram realizadas 61 entrevistas etnográficas, com duração média de 40 min cada e aplicação de um questionário: 30 na Colônia de Pescadores Z-8 e 31 com usuários do arquipélago. A fim de esclarecer aos pescadores que as entrevistas faziam parte de uma pesquisa acadêmica e que as informações não seriam transformadas em produtos comercializáveis, o presidente da Colônia de Pescadores Z-8 assinou uma anuência, permitindo a execução da pesquisa junto à instituição. Antes de cada entrevista, os objetivos da pesquisa foram explicados a cada entrevistado e eles foram questionados se aceitavam participar do estudo. Também, antes de cada entrevista, era explicado que os sobrenomes não seriam anotados e que seria solicitado somente o primeiro nome para que o pesquisador pudesse se comunicar com o entrevistado (Librett \& Perrone 2010).

As entrevistas com o GA foram realizadas na sede da Colônia de Pescadores Z-8 e do GB no Clube Marimbás, Iate Clube de Jurujuba, Marina da Glória e quiosques das Praias de Ipanema e Copacabana. Para o GA, o tamanho amostral representou 33,3\% do total de pescadores registrados na Colônia de Pescadores Z-8. Para o GB, a representatividade amostral não pode ser estimada porque não existe um registro do número de usuários recreacionais para a área. Neste tipo de pesquisa, no entanto, a frequência é raramente importante, pois o principal objetivo é entender a subjetividade relacionada ao conhecimento local (Crouch \& McKenzie 2006). Estudos que envolvem o conhecimento local são subjetivos e complexos, pois são baseados em crenças e simbologias de uma comunidade (Begossi 1992). Nesse sentido, a pesquisa qualitativa não quantifica, mas permite a aproximação entre o sujeito e o objeto, o que possibilita ao pesquisador se aprofundar no contexto cultural. Assim, se a amostra é extensa, a obtenção de mais relatos pode apenas tornar a informação repetitiva (Mason 2010). 


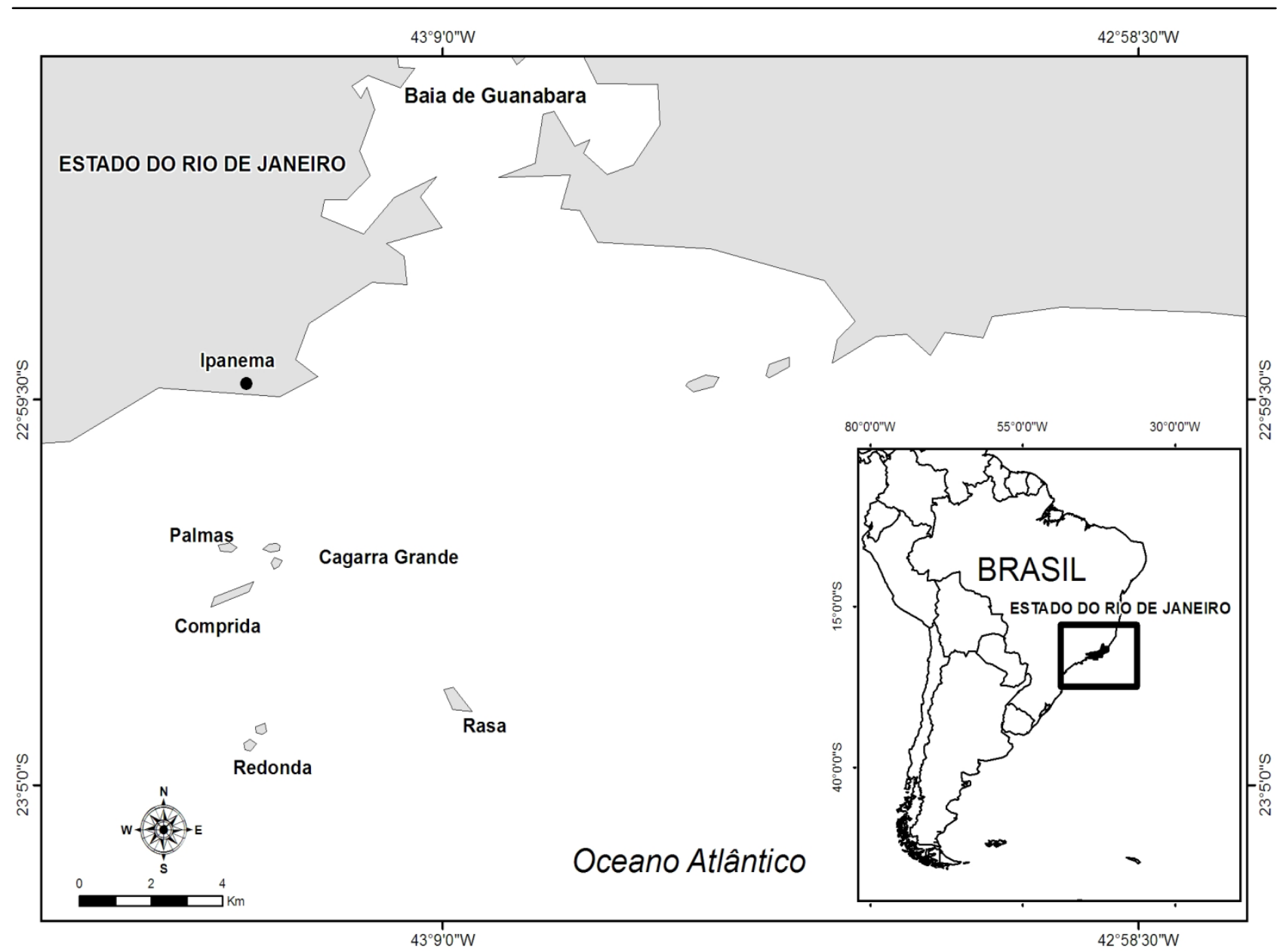

Figura 1. Localização geográfica do Arquipélago das Cagarras, Rio de Janeiro, sudeste do Brasil, com relação à Baía de Guanabara e a Praia de Ipanema.

Em pesquisas relacionadas à ecologia humana, o nível de realidade não pode ser quantificado, pois é considerado um universo de significados, valores e atitudes os quais não podem se reduzidos às variáveis (Kendall 2008). Neste sentido, em estudos sobre percepção local é descrito um tamanho amostral ideal entre 30 e 60 entrevistas (Morse 1994; Bernard 2000). Assim como neste trabalho, outros estudos etnoecológicos e etnobiológicos relacionados à percepção sobre cetáceos também utilizaram tamanhos amostrais equivalentes a menos de $50 \%$ da comunidade estudada: Souza \& Begossi (2007), com aproximadamente cinco entrevistados em cada comunidade em São Sebastião, São Paulo; Peterson et al. (2008), com 51 entrevistas em Laguna, Santa Catarina; Zappes et al. (2009), com 20 entrevistas em cada área estudada na Bahia, Espírito Santo, Rio de Janeiro e São Paulo; Zappes et al. (2010a; 2011a), com 22 entrevistas no Arquipélago das Cagarras; e Zappes et al. (2011b), com 22 entrevistas na Barra de Imbé/Tramandaí, Rio Grande do Sul. Desta forma, o tamanho amostral neste estudo mostra-se ideal para a obtenção de informações robustas relacionadas à percepção local.

A seleção dos entrevistados ocorreu em três etapas: 1- no GA, com auxílio do presidente da Colônia de
Pescadores Z-8, e no GB, com auxílio de um guia, frequentador mais antigo do Arquipélago das Cagarras; 2- para ambos os grupos, foi utilizado o método Bola de neve (Biernacki \& Waldorf 1981; Bailey 1982) ou rede (Bott 1976); e 3- aleatoriedade. Estas etapas são descritas a seguir:

1) No GA, os pesquisadores tiveram a colaboração do presidente da Colônia de Pescadores apenas para a seleção do primeiro entrevistado, já que geralmente é aquele que melhor conhece os pescadores da região (Sanches 2004). No GB, os pesquisadores tiveram a colaboração de um guia - usuário recreacional que frequenta há mais tempo o Clube Marimbás, Iate Clube de Jurujuba, Marina da Glória e quiosques das Praias de Ipanema e Copacabana - apenas para a seleção do primeiro entrevistado.

2) Tanto o presidente da Colônia de Pescadores Z-8 quanto o guia local apresentaram o primeiro possível entrevistado de cada grupo; a partir do segundo entrevistado, os pesquisadores aplicaram o método Bola de neve ou rede, no qual um potencial entrevistado era indicado pelos membros de cada grupo que já haviam respondido ao questionário. Esta técnica trabalha com cadeias de referência para 
o recrutamento, fazendo uso das relações entre as pessoas. Após cada entrevista, foi solicitado ao entrevistado que indicasse outros atores que poderiam participar do estudo e o processo se repetiu com os novos entrevistados, formando uma rede de informantes (Panton 1990). Esse método parte do pressuposto de que é mais fácil um membro do grupo conhecer outros do que os pesquisadores os identificarem.

3) A fím de minimizar a possibilidade de seleção dos entrevistados e tendenciamento das entrevistas, o método Bola de neve ou rede era interrompido e a aproximação ao entrevistado seguinte ocorria de modo aleatório, a partir de encontros oportunísticos.

Como critérios, os entrevistados do GA deveriam: 1- ser pescadores artesanais; 2- ter a pesca como principal atividade econômica; e 3- praticar a pesca artesanal no Arquipélago das Cagarras e áreas adjacentes. E os entrevistados do GB deveriam: 1- ser frequentadores do Arquipélago das Cagarras, ou seja, visitar o arquipélago pelo menos três vezes por semestre, totalizando seis visitas ao ano. A fim de testar a confiabilidade, confrontar as informações e validar os dados, o mesmo questionário foi aplicado a todos os entrevistados (técnica de informações repetidas em situação sincrônica) (Melo 2004; Opdenakker 2006). As entrevistas foram conduzidas individualmente para evitar possíveis interferências nas respostas e através de diálogos (entrevista pessoal ou face a face), em formato de pergunta-resposta, para que fosse estabelecida a confiança entre entrevistador e entrevistado e desta forma aumentar a confiabilidade dos dados (Opdenakker 2006). Os termos utilizados no questionário estavam em conformidade com o vocabulário usual dos entrevistados, e se basearam em Zappes et al. (2011a).

Durante as entrevistas, foi aplicado um questionário padrão (Kendall 2008), contendo questões abertas $(\mathrm{N}=8)$ e fechadas $(\mathrm{N}=6)$ previamente elaboradas (Schensul et al. 1999), divididas em quatro categorias (Quadro 1).

Quadro 1. Categorias dos tópicos das questões abordadas no questionário.

\begin{tabular}{|l|}
\hline 1. Aspectos socioeconômicos \\
Idade \\
Sexo \\
Profissão \\
Período que frequenta o arquipélago \\
2. Aspectos ecológicos e biológicos do golfinho-flíper \\
Período de avistagem na área de estudo \\
Sazonalidade \\
Comportamento \\
Tamanho populacional \\
3. Interações entre os golfinhos-flíper e usuários do \\
arquipélago \\
Conflitos entre humanos e a espécie \\
Possíveis ameaças antrópicas aos golfinhos \\
4. Necessidade ou não da implementação de medidas de \\
monitoramento
\end{tabular}

Para nos certificarmos de que GA e GB conhecem T. truncatus, foi apresentada a cada entrevistado uma prancha ilustrativa contendo fotos de cinco espécies de odontocetos com ocorrência confirmada para o Rio de Janeiro (Hetzel \& Lodi 1993): golfinho-pintado-doatlântico (Stenella frontalis Cuvier, 1829), golfinho-dedentes-rugosos (Steno bredanenis Lesson, 1828), botocinza (Sotalia guianensis van Bénéden, 1864), golfinho-comum (Delphinus spp.) e golfinho-flíper. Esta ferramenta auxiliou os pesquisadores na certificação de que os entrevistados realmente identificam o golfinho $T$. truncatus e que não o confundem com outras espécies (Miranda et al. 2007).

Análise dos dados. Os dados coletados foram agrupados em categorias (relatos referentes à ocorrência da espécie no arquipélago, sazonalidade, comportamento, uso de hábitat, tamanho populacional, ameaças, pressões antrópicas e medidas para a conservação) para classificar os relatos e facilitar a interpretação do conhecimento local sobre T. truncatus (Bogdan \& Bilken 1994).

O tamanho amostral foi de $51 \quad(83,6 \%)$ entrevistados, considerando apenas aqueles que identificaram a espécie corretamente: $47 \%$ do GA $(\mathrm{N}=24)$ e $53 \%$ do $\mathrm{GB}(\mathrm{N}=27)$. A certeza do reconhecimento de $T$. truncatus pelos entrevistados foi obtida selecionando os relatos que identificaram os golfinhos-flíper pela prancha ilustrativa (características morfológicas) e descreveram sua ocorrência no arquipélago entre julho e dezembro (Barbosa et al. 2008; Lodi 2009). A faixa etária do GA variou entre 20 e 76 anos de idade (41,7\% entre 41 e 50 anos), sendo todos do sexo masculino. Já para os selecionados do $\mathrm{GB}$, a idade variou entre 22 e 80 anos $(33,3 \%$ entre 31 e 40 anos), sendo quatro do sexo feminino e os demais do sexo masculino. Para o GA, somente foi descrita a profissão pescador artesanal, já que todos os entrevistados são cadastrados na Colônia de Pescadores Z-8. No GB, houve uma diversidade de profissões, tais como instrutor de mergulho, mergulhador, biólogo, educador ambiental, médico veterinário, advogado, comerciário, autônomo, marinheiro, auxiliar de serviços gerais, publicitário, empresário, engenheiro, economista, analista de sistemas e artista plástico. Dos 27 entrevistados do GB, 66,7\% $(\mathrm{N}=18)$ responderam que praticam mais de uma atividade no arquipélago, que inclui a pesca esportiva com linha $(\mathrm{N}=10$; $19,6 \%)$, caça submarina $(\mathrm{N}=8 ; 15,6 \%)$, lazer/recreação $(\mathrm{N}=19 ; 39,2 \%)$, mergulho livre e autônomo de contemplação $(\mathrm{N}=10 ; 21,5 \%)$ e pesquisa científica $(\mathrm{N}=2 ; 3,9 \%)$. Os entrevistados do GA frequentam o arquipélago num período de 2 a 65 anos e do GB de 4 a 70 anos.

Para as análises dos relatos dos 51 entrevistados selecionados, foi utilizado o método da Triangulação (Teis \& Teis 2006) a fim de cruzar e filtrar as informações coletadas com os métodos aplicados (observação participante, entrevistas-questionários e 
uso das pranchas). Testes de hipóteses foram aplicados com o objetivo de avaliar a existência ou não de diferenças significativas entre os dois grupos estudados. As análises foram realizadas utilizando Mann-Whitney e Qui-quadrado $(p \leq 0,05)$. Ambos os testes foram feitos através do programa STATISTICA 8.0. O teste de Mann-Whitney foi utilizado para comparação dos relatos do GA e GB em relação às questões de sazonalidade, categorias comportamentais descritas, ocorrência de flutuabilidade do tamanho populacional de golfinhos, possível desaparecimento dos golfinhos, existência de ameaças antrópicas e medidas de conservação para a espécie no arquipélago. Para o teste de Qui-quadrado, foi comparada a percepção do GA e GB sobre as causas que influenciam a escolha dos animais pelo uso da área. A comparação estatística entre esses dois grupos foi realizada a fim de se estabelecer as diferenças na percepção de grupos distintos. Espera-se que o GB, por visitar mais esporadicamente o Arquipélago das Cagarras, apresente um conhecimento local ainda incipiente sobre $T$. truncatus quando comparado aos entrevistados do GA, que atuam na área quase que diariamente.

\section{RESULTADOS}

Ocorrência da espécie no arquipélago. De acordo com os relatos dos entrevistados mais velhos do GA e do GB, T. truncatus tem sido avistado no Arquipélago das Cagarras desde 1945 e 1950, respectivamente. Todos os selecionados do GA e $75 \%(\mathrm{~N}=18)$ do GB vêm os animais desde o primeiro ano em que passaram a frequentar o arquipélago. Quatro entrevistados $(57,1 \%)$ do GB relataram ter avistado os golfinhosflíper pela primeira vez num período de 1 a 4 anos após começarem a frequentar o arquipélago, dois $(28,6 \%)$ responderam após 10 anos e um após 28 anos (14,3\%).

Sazonalidade. Quatro (16,7\%) entrevistados do GA e oito $(29,6 \%)$ do GB responderam não haver uma estação predominante de ocorrência entre o inverno (julho a setembro) e a primavera (outubro a dezembro). Para os demais, a primavera foi a estação preferencialmente respondida por ambos os grupos, $60 \%$ e 78,9\%, no GA e GB, respectivamente. Quando comparada a percepção dos pescadores e usuários do arquipélago em relação à sazonalidade de $T$. truncatus na área de estudo não houve diferença significativa $(\mathrm{U}=4,5 ; p=1,000)$.

Comportamento. Para os entrevistados, são seis os principais comportamentos que os animais exibem no Arquipélago das Cagarras, referentes às categorias comportamentais de alimentação (comer/pescar), reprodução (acasalar/namorar), descanso (descansar/se encostar), cuidado parental (ensinar filhote/ arquipélago é usado como creche), socialização (brincar/vida social), deslocamento (deslocar/ passagem). Dois entrevistados do GA e 15 do GB relataram mais de um comportamento. Os comportamentos mais frequentemente citados foram comer/pescar GA $(\mathrm{N}=23 ; 88,5 \%)$ e $\mathrm{GB}(\mathrm{N}=24$; $57,1 \%)$, acasalar/namorar GA $(\mathrm{N}=1 ; 3,8 \%)$ e $\mathrm{GB}$ $(\mathrm{N}=7 ; 16,7 \%)$ e descansar/se encostar GA $(\mathrm{N}=1$; $3,8 \%)$ e $\mathrm{GB}(\mathrm{N}=5 ; 11,9 \%)$ (Tabela 1$)$. Entrevistados do GB apontaram um maior número de categorias comportamentais exibidas por $T$. truncatus no arquipélago quando comparado ao GA. Não houve diferença significativa entre os relatos dos pescadores e frequentadores em relação aos comportamentos de T. truncatus no Arquipélago das Cagarras ( $\mathrm{U}=13,5$; $p=0,159)$.

Uso de hábitat. Com relação a uma possível explicação para a escolha do arquipélago pelos animais, $62,5 \%(\mathrm{~N}=15)$ do GA relataram que ela está relacionada à procura de alimento, enquanto 77,8\% $(\mathrm{N}=21)$ do $\mathrm{GB}$ descreveram por ser um refúgio com águas abrigadas. Três (12,5\%) componentes do GA e sete $(25,9 \%)$ do GB apontaram as duas causas. Quando comparada a percepção dos grupos de entrevistados em relação às causas que influenciam a escolha dos animais pelo uso da área não houve diferença significativa $\left(\chi^{2}=2,66\right.$; g.1. $\left.=1 ; p=0,169\right)$.

Tamanho populacional, ameaças e pressões antrópicas. Um total de 18 (75\%) entrevistados do GA e $13(48,2 \%)$ do GB respondeu que o número de animais tem diminuído (Tabela 2). A maioria dos entrevistados respondeu que existe a possibilidade de desaparecimento dos golfinhos-flíper do arquipélago: GA- 54,2\% $(\mathrm{N}=13)$; GB- 74,1\% ( $\mathrm{N}=20)$. Quando comparada à percepção dos dois grupos para ambas as perguntas, não houve diferenças significativas $(\mathrm{U}=8,00 ; p=0,347) \mathrm{e}$ $(\mathrm{U}=4.00 ; p=0.827)$, respectivamente.

Tabela 1. Categorias comportamentais de Tursiops truncatus exibidas no Arquipélago das Cagarras, Rio de Janeiro, de acordo com a percepção dos entrevistados. GA = pescadores e GB = frequentadores. * Número de citações.

\begin{tabular}{lcccccc}
\hline Categorias & $\mathbf{G A}^{*}$ & $\mathbf{\%}$ & $\mathbf{G B}^{*}$ & $\mathbf{\%}$ & Total* $^{*}$ & \% \\
\hline Comer/Pescar & 23 & 88,5 & 24 & 57,1 & 47 & 69,1 \\
Acasalar/Namorar & 1 & 3,8 & 7 & 16,7 & 8 & 11,8 \\
Descansar/Se encostar & 1 & 3,8 & 5 & 11,9 & 6 & 8,8 \\
Ensinar filhote/Arquipélago usado como creche & - & - & 4 & 9,5 & 4 & 5,9 \\
Brincar/Vida social & - & - & 1 & 2,4 & 1 & 1,5 \\
Deslocar/Passagem & 1 & 3,8 & - & - & 1 & 1,5 \\
Não sabe & - & - & 1 & 2,4 & 1 & 1,5 \\
\hline Total* & $\mathbf{2 6}$ & $\mathbf{9 9 . 9}$ & $\mathbf{4 2}$ & $\mathbf{1 0 0}$ & $\mathbf{6 8}$ & $\mathbf{1 0 0}$ \\
\hline
\end{tabular}


Tabela 2. Relatos dos entrevistados dos grupos GA (pescadores) e GB (frequentadores) em relação ao tamanho atual da população de Tursiops truncatus no Arquipélago das Cagarras, Rio de Janeiro. * Número de citações.

\begin{tabular}{lcccccc}
\hline Tamanho populacional & $\mathbf{G A}^{*}$ & $\mathbf{\%}$ & $\mathbf{G B}^{*}$ & $\mathbf{\%}$ & Total* & $\mathbf{\%}$ \\
\hline Diminuiu & 18 & 75 & 13 & 48,2 & 31 & 60,8 \\
Aumentou & 5 & 20,8 & 6 & 22,3 & 11 & 21,6 \\
Não sabe & - & - & 5 & 18,3 & 5 & 9,8 \\
Existem variações anuais & 1 & 4,1 & 2 & 7,5 & 3 & 5,8 \\
Se mantém estável & - & - & 1 & 3,7 & 1 & 2 \\
\hline Total* & $\mathbf{2 4}$ & $\mathbf{9 9 , 9}$ & $\mathbf{2 7}$ & $\mathbf{1 0 0}$ & $\mathbf{5 1}$ & $\mathbf{1 0 0}$ \\
\hline
\end{tabular}

Dos selecionados do GA, $62,5 \%(\mathrm{~N}=15)$ relataram que não há ameaça antrópica sobre os golfinhos no arquipélago e áreas adjacentes, enquanto $81,4 \%(\mathrm{~N}=$ 22) do GB responderam que esta ameaça existe e que pode influenciar de forma direta ou indireta o desaparecimento dos golfinhos-flíper no arquipélago. Estas ameaças antrópicas foram descritas somente pelos entrevistados do GB, que apontaram mais de uma ameaça, como o "excesso de embarcações recreacionais no arquipélago", que obteve a maior frequência das respostas $(\mathrm{N}=15 ; 29,4 \%)$, seguida pela "poluição das águas eutrofizadas da Baía de Guanabara" ( $\mathrm{N}=10,19,6 \%)$. Artefatos utilizados pela pesca artesanal, como redes de espera, caceio ou caiçara também foram descritos como ameaças aos golfinhos (GA: $\mathrm{N}=7 ; 29,2 \%$ e $\mathrm{GB}: \mathrm{N}=6 ; 11,8 \%$ ) (Tabela 3). Houve diferença significativa entre a percepção dos pescadores e frequentadores em relação às ameaças antrópicas sobre $T$. truncatus no arquipélago $(\mathrm{U}=3,50 ; p=0,02)$.

Medidas para a conservação. As medidas citadas pelos entrevistados para a conservação da espécie no arquipélago encontram-se listadas na Tabela 4. Três (12,5\%) entrevistados do GA e 15 (55,5\%) do GB relataram mais de uma medida. Tanto para o GA $(\mathrm{N}=8 ; 28,6 \%)$ quanto para o $\mathrm{GB}(\mathrm{N}=18 ; 34,6 \%), \mathrm{o}$ manejo da pesca e do turismo, respectivamente, foram as respostas predominantes. Nenhum componente do GA respondeu sobre a questão da fiscalização constante abordada pelo GB. Em relação às respostas obtidas sobre esta questão, "não precisa fazer nada" e "não sabe" foram as respondidas em maior número pelo GA quando comparadas às respostas do GB. Um entrevistado (GA) relatou a questão do tratamento dos efluentes do emissário submarino de Ipanema e despoluição da Baía de Guanabara. Nenhum frequentador (GB) respondeu não saber que medidas podem ser adotadas para a conservação dos golfinhos, enquanto esta resposta foi obtida para seis citações do GB. Apesar dos entrevistados citarem com maior frequência o manejo do turismo $(\mathrm{GA}=21,4 \%$; $\mathrm{GB}=34,6 \%)$ e da pesca $(\mathrm{GA}=28,6 \%$; $\mathrm{GB}=32,7 \%)$ como medidas para a conservação da espécie, não houve diferença significativa entre as respostas dos pescadores e frequentadores sobre as medidas de conservação de $T$. truncatus que devem ser implementadas no Arquipélago Cagarras $(U=13.5$; $p=0.471)$.

\section{DISCUSSÃo}

As informações obtidas através da percepção local reportam os registros mais antigos sobre a ocorrência de T. truncatus no Arquipélago das Cagarras, uma vez que o primeiro registro publicado foi feito em 1993 através de uma avistagem oportunística (Hetzel \& Lodi 1993). Nesse estudo, o objetivo não foi identificar possíveis erros no conhecimento local dos pescadores e usuários recreacionais que utilizam a área do Arquipélago das Cagarras, mas identificar a percepção destes dois grupos a fim de complementar as informações sobre T. truncatus fornecidas pela cultura local e dados científicos (Thomas 1993). A troca de informações entre os membros locais e pesquisadores pode ser complexa, incluindo as diferenças entre os dois tipos de linguagem (cultural e científica) (Foale 2006). Neste sentido, estudos sobre percepção local facilitam a aproximação e troca de informações entre cientistas e grupos sociais (Dale \& Armitage 2011). Essa aproximação permitiu a incorporação de novos conhecimentos sobre o golfinho-flíper no arquipélago.

Tabela 3. Ameaças antrópicas sobre Tursiops truncatus no Arquipélago das Cagarras, Rio de Janeiro, e áreas adjacentes descritas pelos entrevistados. $\mathrm{GA}=$ pescadores e $\mathrm{GB}=$ frequentadores. * Número de citações.

\begin{tabular}{|c|c|c|c|c|c|c|}
\hline Ameaças & GA* & $\%$ & GB* & $\%$ & Total* & $\%$ \\
\hline Nenhuma & 15 & 62,5 & 4 & 7,8 & 19 & 25,3 \\
\hline Rede de espera, malha, caceio ou caiçara & 7 & 29,2 & 6 & 11,8 & 13 & 17,3 \\
\hline Águas poluídas da Baía de Guanabara & - & - & 10 & 19,6 & 10 & 13,3 \\
\hline Excesso de embarcações recreacionais & - & - & 15 & 29,4 & 15 & 20 \\
\hline Excesso de usuários & - & - & 7 & 13,7 & 7 & 9,3 \\
\hline Pesca predatória & - & - & 7 & 13,7 & 7 & 9,3 \\
\hline Diminuição dos recursos alimentares & 2 & 8,3 & 2 & 3,9 & 4 & 5,3 \\
\hline Total* & 24 & 100 & 51 & 100 & 75 & 100 \\
\hline
\end{tabular}


Tabela 4. Medidas de conservação de Tursiops truncatus no Arquipélago das Cagarras, Rio de Janeiro, sugeridas pelos entrevistados dos grupos GA (pescadores) e GB (frequentadores). * Número de citações.

\begin{tabular}{|c|c|c|c|c|c|c|}
\hline Medidas & GA* & $\%$ & GB* & $\%$ & Total & $\%$ \\
\hline Manejo da pesca & 8 & 28,6 & 17 & 32,7 & 25 & 31,3 \\
\hline Manejo do turismo & 6 & 21,4 & 18 & 34,6 & 24 & 30 \\
\hline Não precisa fazer nada & 7 & 25 & 3 & 5,8 & 10 & 12,5 \\
\hline Fiscalização constante & - & - & 9 & 17,3 & 9 & 11,3 \\
\hline Tratamento dos efluentes do emissário submarino e despoluição da Baía de Guanabara & 1 & 3,6 & 5 & 9,6 & 6 & 7,5 \\
\hline Não sabe & 6 & 21,4 & - & - & 6 & 7,4 \\
\hline Total* & 28 & 100 & 52 & 100 & 80 & 100 \\
\hline
\end{tabular}

Os entrevistados do GA relataram, principalmente, que os golfinhos utilizam a área para a alimentação, provavelmente por ser uma atividade comportamental diretamente relacionada à pesca artesanal. Ambos, pescadores e golfinhos, podem buscar o mesmo recurso no Arquipélago das Cagarras: os pescadores capturam o pescado para a comercialização e os golfinhos para alimentação. No entanto, por meio das entrevistas com o GA, não foi identificada a existência desta competição. Assim como neste estudo, Barbosa et al. (2008) reportaram a alimentação como o comportamento executado com maior frequência pelo golfinho-flíper no Arquipélago das Cagarras e Zappes et al. (2010a) descreveram que pescadores também citam a alimentação como o principal comportamento exibido pelo golfinho-flíper para a mesma localidade. Em outras áreas de ocorrência da espécie no litoral brasileiro, a alimentação também foi o comportamento predominantemente registrado (Simões-Lopes 1991; Mattos et al. 2007). Estes animais investem a maior parte do tempo em atividades relacionadas à alimentação (Wells \& Scott 2002), o que justifica para este estudo, a maior frequência de relatos relacionados a este comportamento. $\mathrm{O}$ fato do GB descrever um número maior de comportamentos exibidos por T. truncatus em relação ao GA, provavelmente está relacionado aos entrevistados do GB utilizarem a área para atividades relacionadas ao lazer, sugerindo uma maior disponibilidade à contemplação dos animais do que os entrevistados do GA, que frequentam a área para a prática da pesca artesanal e, com isso, observam os golfinhos oportunisticamente.

A categoria comportamental de "descanso" foi a terceira mais descrita. Segundo Mattos et al. (2007), estudos de comportamento para T. truncatus podem subestimar a frequência de observações do descanso, já que geralmente as observações são realizadas somente no período diurno. Estudos experimentais de T. truncatus em cativeiro (Lyamin et al. 2007) e de telemetria (Mate et al. 1995) reportam que indivíduos da espécie descansam principalmente à noite. Neste estudo, o descanso pode ter sido a terceira categoria mais descrita, pois pescadores e usuários recreacionais que praticam a pesca esportiva frequentam 0 Arquipélago das Cagarras principalmente no período do dia, o que pode dificultar a avistagem do descanso.
Por outro lado, este comportamento pode ter sido observado e descrito por pessoas que frequentam o arquipélago também à noite. Os entrevistados descreveram o cuidado parental aos filhotes, que também foi relatado para T. truncatus no Arquipélago das Cagarras pelos pescadores das Colônias de Pescadores Z-13 e Z-8 (Zappes et al. 2010a). A identificação deste comportamento por observadores não especialistas sugere que eles também entendam de formação de grupo, razão sexual, hierarquia dos indivíduos no grupo e, possivelmente, taxa anual de nascimentos (Zappes et al. 2010a). As categorias de socialização e deslocamento descritas neste estudo também são registradas para outras populações de T. truncatus no país e podem estar relacionadas à reprodução (Mattos et al. 2007). A citação de avistagens de filhotes no Arquipélago das Cagarras pelos entrevistados pode justificar os relatos referentes às categorias comportamentais de socialização e deslocamento.

A presença constante de filhotes nos grupos, reportada por Lodi \& Monteiro-Neto (2012), provavelmente permitiu que os entrevistados do GB elaborassem uma percepção de que o arquipélago tratase um local propício para a reprodução dos golfinhos. A utilização do arquipélago como área de cria de filhotes (Lodi \& Monteiro-Neto 2012) também estaria incluída neste contexto. Em um dos relatos, fica clara esta percepção local, já que um entrevistado do GB descreveu que "o arquipélago é utilizado como uma creche". A geomorfologia do arquipélago propicia a ocorrência de águas calmas e abrigadas, sendo portanto, ideais para a cria de filhotes. De fato, o interior do complexo insular trata-se da área preferencialmente ocupada por T. truncatus (Barbosa et al. 2008).

Os dados obtidos neste estudo indicam que os pescadores e usuários recreacionais do Arquipélago das Cagarras avistam T. truncatus na região há vários anos. Através do empirismo, alguns entrevistados identificam como causas da diminuição populacional as atividades antrópicas realizadas na área. As ameaças reportadas para T. truncatus no Brasil (Barreto 2011) estão de acordo com as mencionadas no arquipélago, tais como: mortalidade acidental em artes de pesca (Zappes et al. 2011a), degradação do ambiente costeiro 
(Lodi \& Monteiro-Neto, 2012), tráfego de embarcações (Lodi \& Monteiro-Neto 2012) e exploração excessiva dos recursos marinhos (ECOMAR/SEAP 2009; Rangel et al. 2007), o que reafirma que a espécie encontra-se sob forte pressão antrópica.

Assim como neste estudo, os pescadores entrevistados por Zappes et al. (2011a) também citaram a pesca artesanal como a atividade responsável pela captura acidental. Entretanto, segundo os autores, a captura acidental não é relatada pelos pescadores artesanais como uma interação negativa da atividade de pesca sobre os golfinhos. O fato dos pescadores utilizarem tais artefatos pode fazer com que não desejem demonstrar que a captura acidental de cetáceos é causada pela pesca artesanal praticada por eles e com isso ficam isentos de qualquer responsabilidade relacionada a esta interação. Capturas acidentais de cetáceos costeiros em equipamentos de pesca ocorrem em larga escala no Brasil, constituindo uma das principais ameaças a esses animais (Di Beneditto 2003).

A diminuição do número de golfinhos ao longo dos anos, como descrito pelos entrevistados deste estudo, corrobora os resultados obtidos por Lodi \& MonteiroNeto (2012), que reportam uma redução do número médio de indivíduos/grupo a partir de 2008. A perda da biodiversidade e/ou degradação do ambiente marinho, além do intenso fluxo de embarcações nos feriados e fins de semana, possivelmente são as causas para a diminuição da população de $T$. truncatus no Arquipélago das Cagarras (Lodi \& Monteiro-Neto 2012). Outra razão de especial preocupação quanto à conservação de $T$. truncatus é o molestamento intencional aos golfinhos pelos usuários recreacionais do arquipélago devido à constância de indivíduos imaturos nos grupos (Lodi \& Monteiro-Neto 2012). Lanchas e jet skis são conduzidos em alta velocidade perseguindo os golfinhos-flíper e são feitas tentativas forçadas de mergulhos com os animais. $\mathrm{O}$ arquipélago também se encontra diretamente afetado por fontes poluidoras, como o Emissário Submarino de Esgotos de Ipanema, que lança uma carga de dejetos não tratados a menos de $2 \mathrm{~km}$ da ponta sul da Ilha de Palmas (Carreira \& Wagener 1998) e por plumas do emissário submarino da Barra da Tijuca (SisBAHIA 2012). O complexo insular também recebe influência das águas eutrofizadas da Baía de Guanabara (Rangel et al. 2007).

Rangel et al. (2007) reportam que o Arquipélago das Cagarras apresenta uma fauna de peixes recifais relativamente diversa quando comparada a outras áreas do sudeste do Brasil. No entanto, a ausência de algumas espécies de peixes recifais, principalmente predadores de topo, sugerem que a área encontra-se sob severo impacto ambiental (Rangel et al. 2007). Em termos gerais, é notável a diminuição na abundância dos recursos pesqueiros no arquipélago e áreas adjacentes quando comparado à produção de 20 anos atrás (ECOMAR/SEAP 2009). Segundo o
ECOMAR/SEAP (2009), pescadores da Colônia Z-8 apontaram que, além dos impactos e conflitos com a pesca industrial e/ou com grandes embarcações, outras duas práticas são consideradas especialmente predatórias: a pesca com redes de arrasto no interior do complexo insular e a pesca subaquática com equipamento de respiração autônoma (pesca de compressor). A pesca de arrasto de fundo com portas ("chifrudos") é feita eventualmente por embarcações provenientes de comunidades do interior da Baía de Guanabara (p. ex., Ilha da Conceição) (ECOMAR/SEAP 2009). A captura e mortalidade de peixes juvenis e outros organismos marinhos sem discriminação é considerada extremamente prejudicial à integridade e sustentabilidade dos recursos naturais do entorno das Ilhas Cagarras (ECOMAR/SEAP 2009). Curiosamente, a pesca predatória não foi mencionada pelos entrevistados do GA dessa mesma colônia como uma ameaça potencial para T. truncatus e sim pelos integrantes do GB. Isso pode estar relacionado ao fato descrito anteriormente, em que não há interesse dos pescadores em expor a existência da pesca indiscriminada e que alguns artefatos de pesca são os responsáveis pela captura acidental de cetáceos.

Os resultados obtidos neste estudo complementam os reportados por Zappes et al. (2010a, 2011a), que verificaram a clara percepção de pescadores artesanais que atuam no Arquipélago das Cagarras e áreas adjacentes sobre os comportamentos de T. truncatus avistados na região e interações com a atividade de pesca. Como este é o primeiro estudo que envolve a percepção de frequentadores do Arquipélago das Cagarras com o perfil do GB, os resultados referentes a este grupo foram descritivos, não sendo possível compará-los com outros trabalhos.

$\mathrm{O}$ fato de algumas embarcações bem como os equipamentos de pesca não estarem de acordo com a legislação vigente pode justificar a ausência de relatos dos pescadores referentes às ações de fiscalização como sugestão de medidas de conservação dos golfinhos. Os próprios entrevistados identificaram preferencialmente como possíveis soluções as duas principais medidas de conservação debatidas no meio acadêmico: o manejo do turismo e o manejo da pesca. Uma ferramenta poderosa para a conservação e monitoramento pode ser implementada quando os conhecimentos científico e popular são usados de modo apropriado e complementar (Daniels \& Vencatesan 1995).

De acordo com Sallenave (1994), um processo de valorização do conhecimento do ator local torna-se difícil e oneroso para planejadores e tomadores de decisões se as comunidades não estiverem envolvidas. Membros locais integrados nos programas de pesquisa e conservação desenvolvem sentimentos de posse pelos projetos, além de sugerirem medidas de manejo tradicionais que podem ser mais vantajosas pelo custo menor e por sua percepção holística sobre o 
ecossistema (Drew 2005). Dessa forma, programas com envolvimento das comunidades locais passam a ter maiores chances de sucesso (Drew 2005).

Apesar de serem grupos distintos, após o cruzamento de saberes dos pescadores (GA) e de outras culturas com diferentes contextos socioculturais (GB), o conhecimento local de ambos aparentemente mostrou-se homogêneo. Isto pode ser explicado pelo fato de que os dois grupos utilizam a mesma área, seja pela obtenção da renda ou pelo lazer, o que permite avistagens de $T$. truncatus. Há ainda a facilidade em se obter informações sobre a espécie, pelos meios de comunicação, como a mídia televisiva, que está disponível para ambos os grupos. Por ser o Arquipélago das Cagarras próximo de um grande centro urbano, existe a divulgação da necessidade da conservação do ambiente. Por atraírem a atenção do público em geral, golfinhos são popularmente considerados carismáticos e, neste sentido, são explorados pela mídia que expõe vários programas com informações sobre as espécies. Segundo Drews (2002), quanto maior a empatia sobre um dado elemento, maior deverá ser a probabilidade do observador relacionar-se com ele e prover informações. Essa empatia com T. truncatus foi constatada por Zappes et al. (2010a) em entrevistas com pescadores que atuam no Arquipélago das Cagarras, onde alguns entrevistados atribuíram "qualidades humanas" aos golfinhos. A experiência do contato diário, facilitado pela prática da pesca artesanal no arquipélago, permitiu que os pescadores elaborassem e acumulassem um conhecimento local detalhado sobre $T$. truncatus (Zappes et al. 2010a).

Estudos etnobiológicos realizados por Zappes et al. (2010a, 2011a) reportam que os dados obtidos através do conhecimento local podem contribuir na elaboração de planos de gestão e manejo dos recursos naturais em Unidades de Conservação e nas áreas adjacentes, como é o caso do referido arquipélago. $\mathrm{O}$ conhecimento local sobre a espécie se torna mais robusto a partir do momento em que informações etnobiológicas e da literatura científica se complementam (Zappes et al. 2010a). Existe ainda o fato de que a aproximação entre pesquisadores e a comunidade local possa auxiliar no monitoramento de atividades realizadas no local e dos cetáceos encalhados ou capturados acidentalmente (Zappes et al. 2011a).

Recomendações. Com o objetivo de auxiliar o manejo da população de $T$. truncatus que ocorre no Arquipélago das Cagarras, são sugeridas propostas de ações iniciais voltadas à conservação e políticas públicas em que poderão participar tanto atores locais, instituições de pesquisas, organizações nãogovernamentais e governamentais:

1. Adaptar o turismo já existente para um turismo sustentável e/ou comunitário de observação da biota marinha utilizando $T$. truncatus como espécie- bandeira. Esta alternativa pode ser economicamente viável aos pescadores artesanais da região, já que poderia gradualmente substituir a atividade de pesca artesanal durante a temporada de ocorrência dos golfinhos no arquipélago.

2. Promover estudos de mercado para esta atividade turística com a participação dos atores locais e de profissionais das áreas de turismo, economia, administração, biologia e oceanografia.

3. Elaborar protocolos de capacidade suporte de embarcações e para as atividades de observação dos golfinhos-flíper.

4. Qualificar a comunidade pesqueira e frequentadores através de cursos de capacitação de gerência empresarial do turismo sustentável bem como cursos voltados para a conservação de ecossistemas marinhos.

5. Realizar estudos de longo prazo voltados para a Ecologia Humana de forma que se entenda a percepção local sobre o Arquipélago das Cagarras, possibilitando pesquisadores e ações de políticas públicas utilizarem uma linguagem acessível aos atores locais e aplicarem o conhecimento ecológico local na execução de manejos tradicionais de baixo custo para a prática da pesca artesanal e do turismo sustentável.

6. Incentivar a mobilização social na gestão do Monumento Natural do Arquipélago das Ilhas Cagarras, através de seu conselho gestor, promovendo oportunidades para que as comunidades locais e demais envolvidos tenham um melhor entendimento do tema na participação de ações de conservação para os golfinhos-flíper.

7. Realizar levantamento dos artefatos, embarcações e áreas utilizadas na pesca artesanal praticada no Arquipélago das Cagarras e áreas adjacentes, bem como registrar os períodos do ano em que cada tipo de pescaria é praticada.

8. Regular e fiscalizar as atividades de pesca artesanal no Arquipélago das Cagarras e áreas adjacentes.

9. Propor modificações físicas nos artefatos, como a instalação de equipamentos acústicos que emitem frequências de acordo com o sistema de audição dos animais a fim de tornar estes materiais perceptíveis a $T$. truncatus e com isso tentar diminuir os acidentes envolvendo a espécie e a atividade de pesca.

Considerações finais. Os resultados obtidos neste estudo por meio de entrevistas com pescadores e usuários recreacionais contribui para o melhor entendimento de questões ainda não respondidas. Por exemplo, as avistagens mais antigas datam de mais de 60 anos atrás. Esta informação pode auxiliar na compreensão do período de uso do hábitat e se atividades antrópicas atuais podem interferir na viabilidade da manutenção dos golfinhos-flíper no arquipélago. É admissível que as atividades recreacionais realizadas há mais de 60 anos atrás sejam 
diferentes das atuais, assim como o tráfego de embarcações e a pesca, possivelmente, mais intensos atualmente. O conhecimento local dos entrevistados sobre sazonalidade, comportamento, uso do hábitat, tamanho populacional, ameaças e pressões antrópicas coincidem com as informações descritas na literatura. A junção de dados obtidos empiricamente e pelo método tradicional científico pode se tornar útil a partir do momento que essas duas fontes forneçam informações complementares. Os pescadores artesanais e usuários recreacionais são capazes de identificar possíveis impactos antrópicos e a necessidade da implementação de medidas de conservação e monitoramento para os golfinhos-flíper. No entanto, existem diferenças entre a percepção dos pescadores e a dos usuários recreacionais em relação às ameaças antrópicas. Ações iniciais para direcionar medidas de conservação para $T$. truncatus, além da manutenção da pesca artesanal e do turismo sustentável, podem contribuir para as regras de ordenamento e normas de uso de zoneamento do Plano de Manejo do Monumento Natural do Arquipélago das Ilhas Cagarras.

\section{AgRADECIMENTOS}

À CAPES pela concessão da bolsa de pósdoutorado à C.A. Zappes (Processo 87414) e à FAPERJ (E-26/102.708/2011). Aos pescadores artesanais e frequentadores do Arquipélago das Cagarras, que gentilmente colaboraram com as entrevistas, e a Sérgio Carvalho Moreira, pela elaboração da Figura 1.

\section{REFERÊNCIAS}

Bailey, K.D. 1982. Methods of Social Research. Macmillan, Nova York.

Barbosa, M.M.C.; Cruz, F.S. \& Lodi, L. 2008. Comportamento e organização de grupo do golfinho-flíper, Tursiops truncatus (Cetacea, Delphinidea) no arquipélago das Cagarras, Rio de Janeiro. Revista Brasileira de Zoociências 10: 213-220.

Barreto, A.S. 2011. Golfinho-nariz-de-garrafa. In: C.C. RochaCampos, I.G. Câmara \& D.J. Pretto (orgs), Plano de Ação Nacional para a Conservação dos Mamíferos Aquáticos: pequenos cetáceos. Instituto Chico Mendes de Conservação da Biodiversidade. Diretoria de Pesquisa, Avaliação e Monitoramento da Biodiversidade Coordenação Geral de Manejo para a Conservação/ICMBio, Brasília, p. 27-29.

Begossi, A. 1992. Food taboos at Búzios Island (Brazil): their significance and relation to folk medicine. Journal of Ethnobiology 12: 117-139.

Bernard, H.R. 2000. Social Research Methods: qualitative and quantitative approaches. Sage Publications, Thousand Oaks.

Biernacki, P. \& Waldorf, D. 1981. Snowball sampling. Problems and techniques of chain referral sampling. Sociological Methods and Research 10: 141-163.
Bogdan, R.C. \& Bilken, S.K. 1994. Investigação Qualitativa em Educação. 2 ed. Porto Editora, Porto.

Bott, E. 1976. Família e Rede Social. 2 ed. Francisco Alves Editora S/A, Rio de Janeiro.

Carreira, R.S. \& Wagener, A. L. 1998. Speciation of sewage derived phosphorus in coastal sediments from Rio de Janeiro, Brazil. Marine Pollution Bulletin 36: 818-827.

Crouch, M. \& McKenzie, H. 2006. The logic of small samples in interview-based qualitative research. Social Science Information 45: 483-499.

Dale, A. \& Armitage, D. 2011. Marine mammal co-management in Canada's Arctic: knowledge co-production for learning and adaptive capacity. Marine Policy 35: 440-449.

Daniels, R.J.R. \& Vencatesan, J. 1995. Traditional ecological knowledge and sustainable use of natural resources. Current Science 69: 569-570.

Di Beneditto, A.P.M. 2003. Interactions between gillnet fisheries and small cetaceans in northern Rio de Janeiro, Brazil: 20012002. The Latin American Journal of Aquatic Mammals 2: 7986.

Diegues, A.C. 2001. As populações humanas em Áreas Naturais Protegidas. In: A.C. Diegues (ed.), Ecologia Humana $e$ Planejamento Costeiro. 2 ed. Nupaub, São Paulo, p. 169-184.

Drew, J.A. 2005. Use of traditional ecological knowledge in marine conservation. Conservation Biology 19: 1286-1293.

Drews, C. 2002. Attitudes, knowledge and wild animals as pets in Costa Rica. Anthrozoös 2: 119-138.

ECOMAR/SEAP (Secretaria Especial de Aquicultura e Pesca) 2009. Caracterização da atividade pesqueira no entorno das Ilhas Cagarras, Rio de Janeiro - RJ: informações para a criação de uma unidade de conservação. Convênio SEAP No 045/07. Processo No 00.350.003405/2007-12.

Foale, S. 2006. The intersection of scientific and indigenous ecological knowledge in coastal Melanesia: implications for contemporary marine resource management. International Social Science Journal 58: 129-137.

Fruet, P.F.; Kinas, P.G.; Silva, K.G.; Di Tullio, J.C.; Monteiro, D.S.; Dalla Rosa, L.; Estima, S. E \& Secchi, E.R. 2010. Temporal trends in mortality and effects of by-catch on common bottlenose dolphins, Tursiops truncatus, in southern Brazil. Journal of the Marine Biological Association of the United Kingdom 91: 1-12.

Fundação de Amparo à Pesquisa de Recursos Vivos na Zona Econômica Exclusiva. 2005. Relatório técnico sobre o censo estrutural da pesca artesanal marítima e estuarina nos estados do Espírito Santo, Rio de Janeiro, Paraná, Santa Catarina e Rio Grande do Sul. In: J.B. Silva-Filho, S.N. Bezerra \& J.A.N. Aragão (Coords). Fundação PROZEE/ SEAP, PR/IBAMA, Itajaí.

Hetzel, B. \& Lodi, L. 1993. Baleias, Botos e Golfinhos: guia de identificação para o Brasil. Editora Nova Fronteira, Rio de Janeiro.

IBAMA (Instituto Brasileiro do Meio Ambiente e dos Recursos Naturais Renováveis) 2001. Mamíferos Aquáticos do Brasil: plano de ação, versão II. Diretoria de Fauna e Recursos Pesqueiros/IBAMA, Brasília.

Kendall, L. 2008. The conduct of qualitative interview: research questions, methodological issues, and researching online. In: J. 
Coiro, M. Knobel, C. Lankshea \& D. Leu (eds), Handbook of Research on New Literacies. Lawrence Erlbaum Associates, New York, p. 133-149.

Librett, M. \& Perrone, D. 2010. Apples and oranges: ethnography and the IRB. Qualitative Research 10: 729-747.

Lodi, L. 2009. Fidelidade de Área, Características de Grupo e Organização Social de Tursiops truncatus (Cetacea, Delphinidade) no Arquipélago das Cagarras, Rio de Janeiro, RJ, Brasil. Tese de Doutorado. Universidade Federal Fluminense.

Lodi, L. \& Monteiro-Neto, C. 2012. Group size and composition of Tursiops truncatus (Cetacea: Delphinidae) in a coastal insular habitat off southeastern Brazil. Biotemas 25: 157-164.

Lyamin, O.; Pryaslova, J.; Kosenko, P. \& Siegel, J. 2007. Behavioral aspects of sleep in bottlenose dolphin mothers and their calves. Physiology \& Behavior 92: 725-733.

Mason M. 2010. Sample size and saturation in $\mathrm{PhD}$ studies using qualitative interviews. Forum Qualitative Sozialforschung/Forum Qualitative Social Research 11. Disponível em <http://www.qualitative-research.net/index.php/ fqs/article/view/1428>; acesso em 21 mar. 2012.

Mate, B.R.; Rossbach, K.A.; Nieukirk, S.L.; Wells, R.S.; Irvine, A.B.; Scott, M.D. \& Read, A.J. 1995. Satellite-monitored movements and dive behavior of a bottlenose dolphin (Tursiops truncatus) in Tampa Bay, Florida. Marine Mammal Science 11: 452-463.

Mattos, P.H.; Dalla Rosa, L. \& Fruet, P.F. 2007. Activity budgets and distribution of bottlenose dolphins (Tursiops truncatus) in the Patos Lagoon estuary, Southern Brazil. The Latin American Journal of Aquatic Mammals 6: 161-169.

Melo, L.G. 2004. Antropologia cultural, objetivo e método. In: L.G. Melo (ed.), Antropologia Cultural: iniciação, teoria e temas. 11 ed. Editora Vozes, Petrópolis, p. 33-78.

Miranda, T.M.; Amorozo, M.C.M.; Govone, J.S. \& Miranda, D.M. 2007. The influence of visual stimuli in ethnobotanical data collection using the listing task method. Field Methods 19: $76-86$.

Moraes, F.; Bertoncini, A. \& Aguiar, A. (eds) 2013. História, Pesquisa e Biodiversidade do Monumento Natural das Ilhas Cagarras. Série Livros $\mathrm{N}^{\circ} 48$. Museu Nacional, Rio de Janeiro.

Morse, J.M. 1994. Designing funded qualitative research. In: N.K. Denzin \& Y.S. Lincoln (eds), Handbook of Qualitative Research. 2 ed. Sage Publications, Thousand Oaks, p. 220-235.

Oliveira, F.; Becatto, M.A.B.; Nordi, N. \& Monteiro-Filho, E.L.A. 2008. Etnobiologia: interfaces entre os conhecimentos tradicional e científico. In: E.L.A. Monteiro-Filho \& K.D.K.A. Monteiro (orgs), Biologia, Ecologia e Conservação do Botocinza (Sotalia guianensis). Edições IBAMA, Brasília, p. 233 261.

Opdenakker, R. 2006. Advantages and disadvantages of Four Interview Techniques in qualitative research. Forum Qualitative Sozialforschung / Forum: Qualitative Social Research 7. Disponível em <http://nbn-resolving.de/urn:nbn:de:0114fqs0604118>; acesso em 21 mar. 2012.

Panton, M.Q. 1990. Qualitative Evaluation and Research Methods. 2 ed. Sage Publications, Newbury Park.

Peterson, D.; Hanazaki, N. \& Simões-Lopes, P.C. 2008. Natural resource appropriation in cooperative artisanal fishing between fishermen and dolphins (Tursiops truncatus) in Laguna, Brazil. Ocean \& Coastal Management 51: 469-475.

Pinheiro, L. \& Cremer, M.J. 2003. Etnoecologia e captura acidental de golfinhos (Cetacea: Pontoporiidae e Delphinidae) na Baía da Babitonga, Santa Catarina. Desenvolvimento e Meio Ambiente 8: 69-75.

Rangel, C.A.; Chaves, L.C. \& Monteiro-Neto, C. 2007. Baseline assessment of the reef fish assemblage from Cagarras Archipelago, Rio de Janeiro, Southeastern Brazil. Brazilian Journal of Oceanography 55: 7-17.

Richardson, R.J. 1999. Pesquisa Social: métodos e técnicas. Editora Atlas, São Paulo.

Sallenave, J. 1994. Giving traditional ecological knowledge its rightful place in environmental impact assessment. Northern Perspectives 22: 16-18.

Sanches, R.A. 2004. Caiçaras e a Estação Ecológica de JuréiaItatins. Histórico de ocupação no contexto político, econômico, social e ambiental do Vale do Ribeira. In: O.A.V. Marques \& W. Duleba (eds), Estação Ecológica Juréia-Itatins. Ambiente fisico, flora e fauna. Holos Editora, Ribeirão Preto, p. 349-359.

Schensul, S.L.; Schensul, J.J. \& LeCompte, M.D. 1999. Essential ethnographic methods: observations, interviews, \& questionnaires. In: J.J. Schensul \& M.D. LeCompte (eds), Ethnographer's Toolkit. 2 ed. Altamira Press, Walnut Creek, p. 69-89.

Simões-Lopes, P.C. 1991. Interaction of coastal populations of Tursiops truncatus (Cetacea, Delphinidae) with the mullet artisanal fisheries in Southern Brazil. Biotemas 2: 83-94.

SISBAHIA (Sistema de Base Ambiental de Hidrodinâmica Ambiental) 2012. Exemplos de resultados. Disponível em < http://www.sisbahia.coppe.ufrj.br>; acesso em 10 maio 2012.

Souza S.P. \& Begossi, A. 2007. Whales, dolphins or fishes? The ethnotaxonomy of cetaceans in São Sebastião, Brazil. Journal of Ethnobiology and Ethnomedicine. Disponível em $<\mathrm{http}: / /$ www.ethnobiomed.com/content/3/1/9>; acesso em 10 maio 2012.

Teis, M.A. \& Teis, D.T. 2006. A abordagem qualitativa: a leitura no campo de pesquisa. Biblioteca On-line de Ciências da Comunicação 1: 1-8. Disponível em <http://bocc.ubi.pt/_esp/ autor.php?codautor=969>; acesso em $10 \mathrm{dez} .2011$.

Thomas, J. 1993. Doing Critical Ethnography. Sage Publications, Thousand Oaks.

Wells, R.S. \& Scott, M.D. 2002. Bottlenose dolphins. In: W.F. Perrin, B. Würsing \& J.G.M. Thewissen (eds), Encyclopedia of Marine Mammals. Academic Press, San Diego, p. 122-128.

Zappes, C.A.; Andriolo, A.; Oliveira, F. \& Monteiro-Filho, E.L.A. 2009. Potential conflicts between fishermen and Sotalia guianensis (van Bénéden, 1864) (Cetacea, Delphinidae) in Brazil. Sitientibus série Ciências Biológicas 9: 208-214.

Zappes, C.A.; Gatts, C.E.N.; Lodi, L; Andriolo, A. \& Di Beneditto, A.P.M. 2010a. Descrição do comportamento do golfinho-nariz-de-garrafa (Tursiops truncatus Montagu, 1821) através da etnoecologia da Lógica Fuzzy. Revista Brasileira de Zoociências 12: 291-304.

Zappes C.A.; Monteiro-Filho E.L.A.; Oliveira, F. \& Andriolo, A. 2010b. O comportamento do boto-cinza Sotalia guianensis (van Bénéden, 1864) (Cetacea; Delphinidae) através do olhar dos pescadores artesanais. Revista de Etologia 1: 7-28. 
Zappes, C.A.; Gatts, C.E.N.; Lodi, L; Andriolo, A. \& Di Beneditto, A.P.M. 2011a. Interações entre o golfinho-nariz-degarrafa (Tursiops truncatus) e a pesca artesanal no Arquipélago das Cagarras e áreas adjacentes, Rio de Janeiro, Brasil. Sitientibus série Ciências Biológicas 11: 4-30.
Zappes, C.A.; Andriolo, A.; Simões-Lopes, P.C. \& Di Beneditto, A.P.M. 2011b. 'Human-dolphin (Tursiops truncatus Montagu, 1821) cooperative fishery' and its influence on cast net fishing activities in Barra de Imbé/Tramandaí, Southern Brazil. Ocean \& Coastal Management 54: 427-432. 